\title{
Emergency reconstruction of large general hospital under the perspective of new COVID-19 prevention and control
}

\author{
Yiyi Chen $(\mathbb{D} \cdot$ Min Zhou $\cdot$ Liang Hu $\cdot$ Xiaoyu Liu $\cdot$ Lixin Zhuo $\cdot$ Qiong Xie
}

Received: 31 March 2020 / Accepted: 28 May 2020 / Published online: 29 June 2020

(C) Springer-Verlag GmbH Austria, part of Springer Nature 2020

\begin{abstract}
Summary
Objective To summarize the successful experience of timely crisis management, correct measures, and successful display of the hospital image in the First Affiliated Hospital of Zhejiang University (FAHZU), to improve the ability of emergency response.

Methods The FAHZU, as the earliest designated hospital, accomplished the transformation from general hospital to infectious disease hospital under the guiding ideology of centralized patients, centralized
\end{abstract}

The authors Yiyi Chen, Min Zhou and Liang Hu contributed equally to this work.

Availability of data and material The datasets analyzed during the current study are not publicly available due to confidentiality reasons but are available from the corresponding author on reasonable request.

Y. Chen $(\bowtie) \cdot$ L. Hu $\cdot$ X. Liu $\cdot$ L. Zhuo

General management office, The First Affiliated Hospital,

Zhejiang University School of Medicine, 79 Qingchun

Road, 310003 Hangzhou, Zhejiang, China

chenyiyi975022@sina.com

L. $\mathrm{Hu}$

zyyyhL@sina.com

X. Liu

xiaoyu3761231@126.com

L. Zhuo

zyzhuolixin@126.com

M. Zhou $\cdot$ Q. Xie

Information Centre, The First Affiliated Hospital,

Zhejiang University School of Medicine, 79 Qingchun

Road, 310003 Hangzhou, Zhejiang, China

M. Zhou

zyzm1993@163.com

Q. Xie

youzi_116@126.com experts, centralized resources, and centralized treatment with measures to transfer the Zhijiang campus hospitalized patients quickly, complete the space layout, create diagnosis and treatment space, streamline logistics, and transform logistics facilities within $48 \mathrm{~h}$. As of 5 March, the hospital had admitted 104 patients. Results Of the severe cases in Zhejiang province 95\% underwent centralized treatment with the goal of zero deaths for severely ill patients, zero misdiagnoses for infected patients, and zero infections for medical staff, and this served as a reference for large medical institutions regarding how to manage such a public health emergency.

Conclusion The successful cases of FAHZU provided a valuable experience for large medical institutions on how to address public health emergencies and how to carry out diagnosis and treatment and streamline the layout and related facilities in emergency reconstruction.

Keywords Coronavirus disease 2019 - Public health emergency · General hospital · Emergency response · Infectious disease hospital

\section{Abbreviations \\ COVID-19 Coronavirus disease 2019 \\ FAHZU First Affiliated Hospital of Zhejiang Uni- versity}

\section{Background}

Beginning in December 2019, a series of unexplained pneumonia cases were reported in Wuhan, China. The coronavirus disease 2019 (COVID-19) has spread worldwide [1-4]. Due to the widespread population susceptibility, a large number of infected patients appeared in a short period of time in China. As the most economically developed province in China, 
Zhejiang has hundreds of thousands of people who travel to Wuhan for business and work every year. Therefore, from the beginning of the epidemic, Zhejiang paid high attention to COVID-19, and initiated a first-level response nationwide at the earliest time to transfer suspected and confirmed cases to designated hospitals for unified treatment. On 19 January 2020, the First Affiliated Hospital of Zhejiang University (FAHZU) admitted the first suspected case from Hangzhou. It was identified as the only provincial fixed-point hospital and the leader of COVID19 diagnosis and treatment, and took charge of the centralized treatment task of severely ill provincial patients. The FAHZU became the main battlefield for epidemics and the most arduous medical institution to undertake the prevention and control of outbreaks. The hospital actively mobilized resources, established a special work organizational structure, and expanded the number of fever clinics and isolation wards in the main hospital area. The number of fever clinics was expanded from 2 to 8 , isolation wards were expanded from 1 to 4 floors, and negative pressure wards were increased from 4 to 59 beds; however, the main campus of the hospital was located in the city center with limited expansion space, the population was dense, and the streamline transformation of people and logistics was difficult. Consequently, the hospital launched an emergency plan to transform the new campus (Zhijiang campus) into a hospital for admission of suspected and confirmed cases to prevent insufficient treatment space in the later period. The main campus was reserved exclusively as a fever clinic. If patients were screened and suspected or confirmed to have COVID-19, they were escorted to the Zhijiang campus by professional medical staff for isolation, observation, and treatment.

\section{General situation of the FAHZU Zhijiang campus}

The FAHZU Zhijiang campus is a newly built general hospital covering an area of $99,990 \mathrm{~m}^{2}$, with a con- struction area of $179,000 \mathrm{~m}^{2}$, and with 1000 beds. The FAHZU Zhijiang campus opened on 1 November 2019. Considering the favorable hardware conditions, convenient surrounding traffic, and distance from the downtown area with a dense population, it not only centralized the medical power and resources of the entire province for the treatment of patients, but also established hospital infection control and material logistic concentration by transferring all patients, including severe cases, suspected cases, and medical staff in close contact with patients, to the Zhijiang campus for isolation treatment and medical observation.

The Zhijiang campus closed the original outpatient service area, transferred the hospitalized patients, and completed emergency reconstruction for wards, the ICU, radiology department, emergency treatment, the fever clinic, and other areas within $48 \mathrm{~h}$ after receiving the mission for admitting COVID-19 patients.

\section{Regional management of different objects}

Management of confirmed patients, suspected patients, medical staff, and logistic support management personnel was done regionally (Fig. 1). The Zhijiang campus transformed the emergency center located in the outpatient service area of Building No. 1 into a fever clinic; severe patients were admitted to the intensive care unit (ICU) in Building No. 5 (58 beds); confirmed common patients were admitted to double rooms in floors 7-9 of Building No. 3 (132 beds); patients suspected of infection were admitted to single rooms on floors 5 and 6 of Building No. 3 (30 beds). In addition, considering the different requirements for beds and emergency facilities during different periods of infectious disease outbreaks, we also reserved floors 3 and 4 of Building No. 3 (88 beds) for reserve rooms.

Due to the highly infectious nature of severe acute respiratory syndrome coronavirus 2 (SARS-CoV-2), it is very important to protect and isolate first-line med-

Fig. 1 Layout and function of each building on the Zhijiang campus

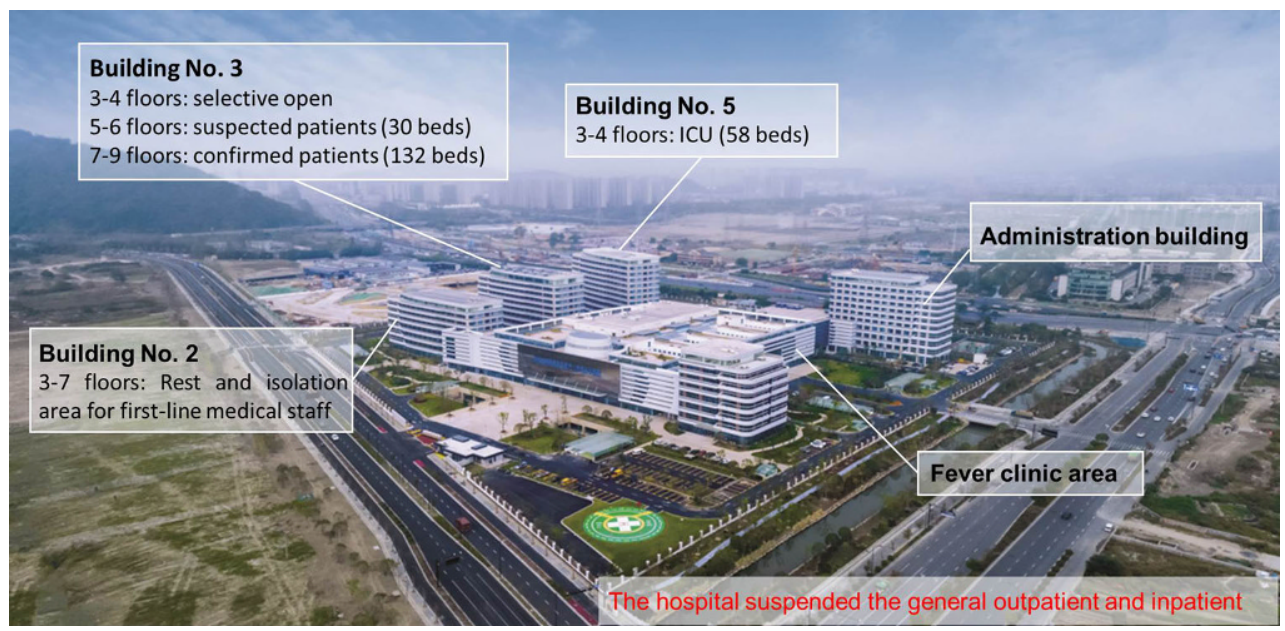



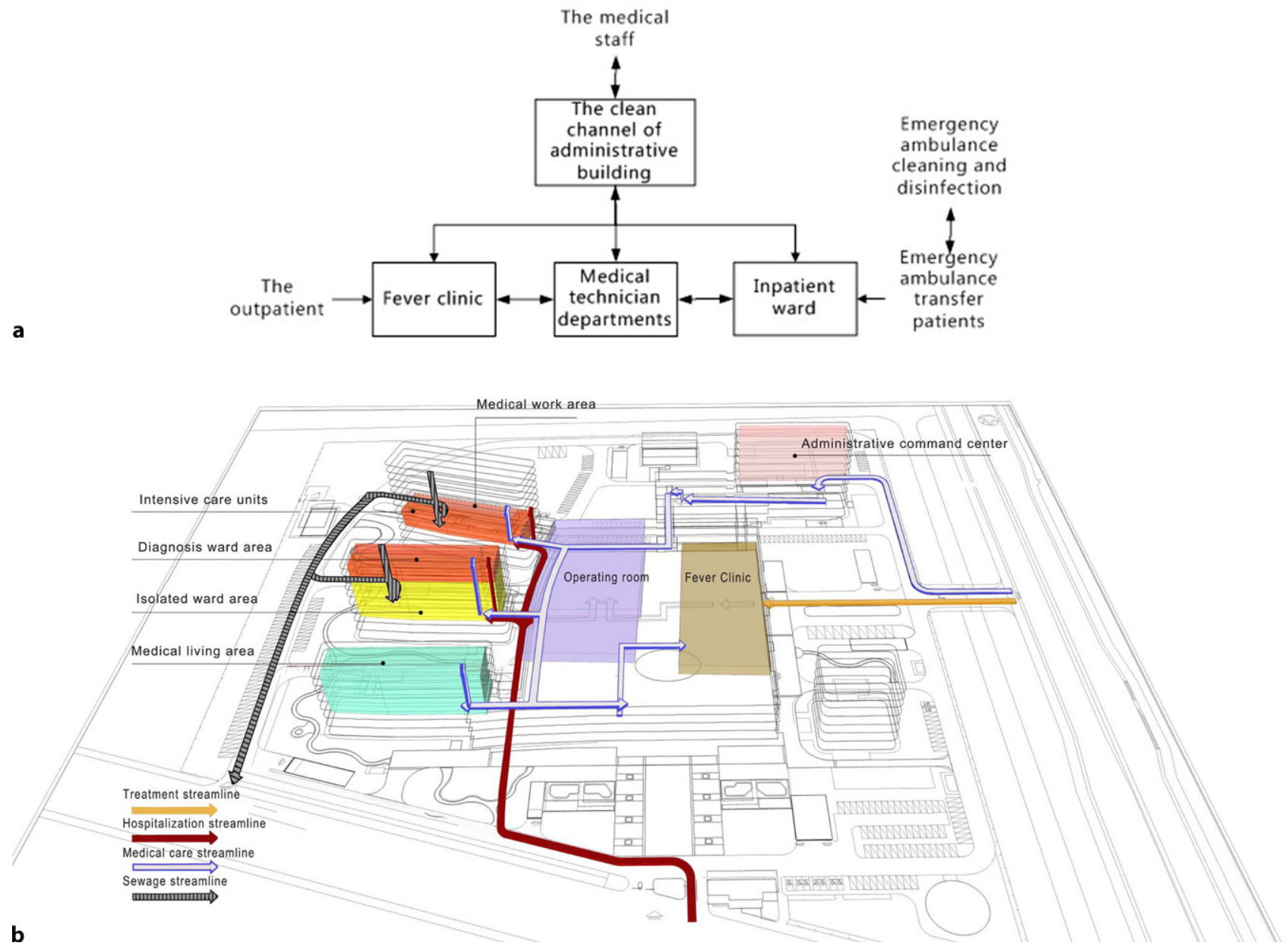

Fig. 2 a Routes of outpatients. b Routes of different objects

ical staff. To ensure the safety of first-line medical staff, the hospital designated the independent Building No. 2 as the medical staff living area, and all medical staff resided in Building No. 2 in isolation during the period of treatment. For the sake of creating a comfortable place for the medical staff to have enough rest and better physical strength and energy to devote to the treatment of patients, the dietary department of the hospital provided them with catering, the material support department provided them with daily necessities and entertainment equipment.

\section{Distribution management of different objects}

The first floor of the ward served as a transfer channel for patients. Patients with fevers entered the emergency center on the first floor of the outpatient building through the east gate, then went to the consulting rooms in the fever clinic. Patients transferred by ambulance from other hospitals in the province entered from the south gate into each ward through the patient elevator on the first floor. It was realized that separation of people and vehicles, as well as the sepa- ration of confirmed patients and patients with general fevers to be screened was necessary (Fig. 2a).

The second floor of the entire hospital served as a clean channel. The medical staff entered into the treatment areas from the clean channel of the second floor of the Administration Building No. 6. In addition, according to the actual situation, the hospital formulated the medical garbage collection and transportation routes, household garbage collection and transportation routes, food delivery routes, and bedding and clothing transportation routes to avoid route crossing (Fig. 2b).

\section{Isolation transformation of the wards}

A plan was adopted to separate the confirmed and suspected patients from floor to floor, with the confirmed patients in a double room and the suspected patients in a single room, thus minimizing the risk of cross-infection. In accordance with the requirements of 3 zones with 2 channels (contaminated area, semicontaminated area, clean area, two buffer zones, patients channels, and employee channels), wards carried out partition transformation for each layer to 


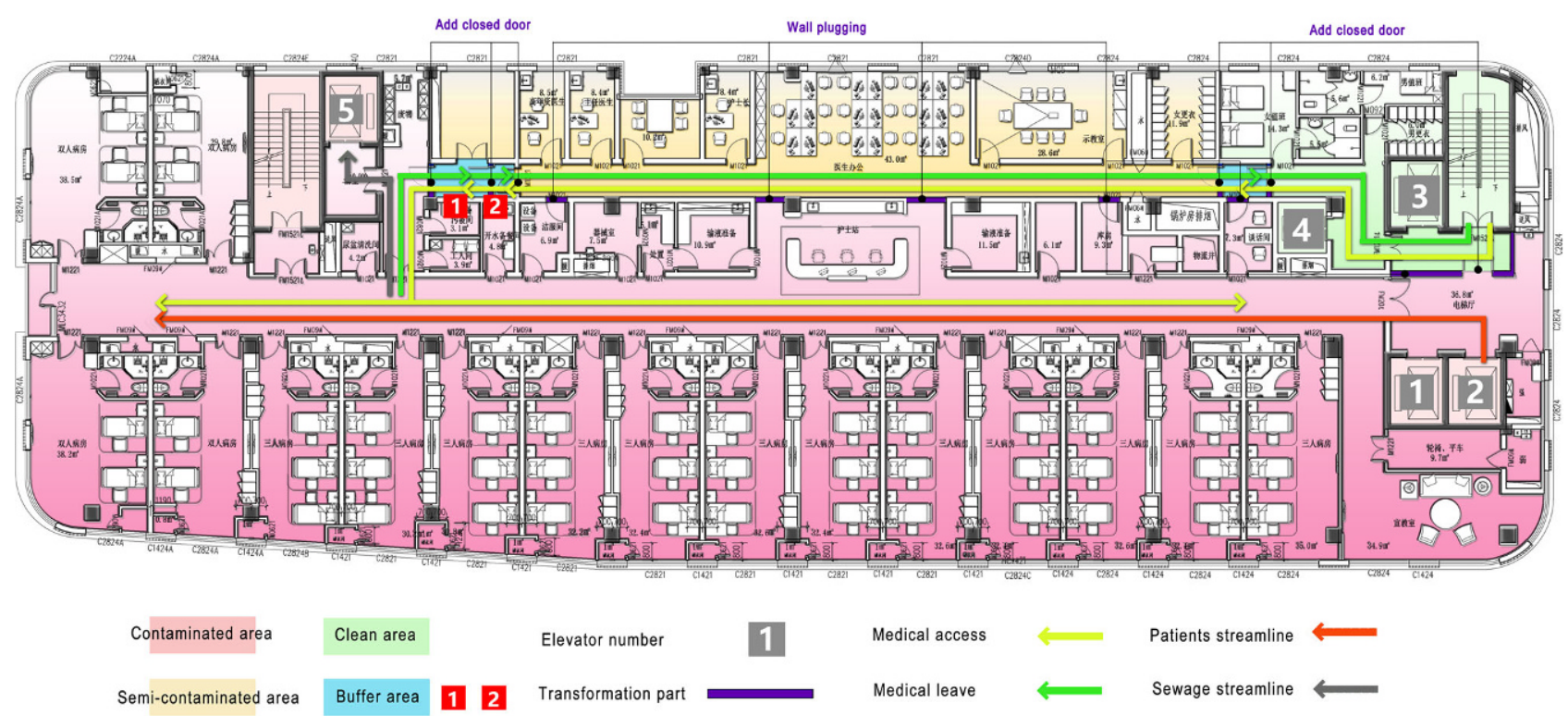

Fig. 3 The streamlining of ward medical staff and patients

separate the physician-patient entrances and exits. There was an explicit distinction between the clean (work and rest for the physician), semi-contaminated (physicians' working channel), and contaminated areas (patient area). The specific practice was as follows: partitions were added on both sides of the nurse station; two partitions were added to transform two buffer zones west of the physician channel; a partition was added for the clean channel east of the physician channel; and the safe channel was separated in the entrance hall. Through the physical partition to achieve the effect of a partition, a buffer space existed between the districts to set up a hand sanitizing device. The streamline of medical staff and patients in the wards is shown in Fig. 3.

\section{Transformation of the fever clinic}

The original emergency center was redesigned with partitions and channel streamlining, and the emergency center was transformed into a fever clinic. The specific transformation plan was as follows: three partitions were made in the northwest side of the nurses' station for the passage of medical staff; and the semicontaminated area and buffer zone were separated. The medical staff entered into the clean area from east of the medical staff channel, and entered the buffer room after donning hats, masks, gloves, goggles, and protective clothing in a clean area, then passed into clinic rooms from the semi-contaminated area. When the medical staff left, they had to change their outer gloves in the contaminated area and take off their outer gloves, protective clothing, goggles, cap, mask, and inner gloves in the semi-contaminated area, then turn into the buffer room to bathe, change clothes, and finally enter the clean area. Closed doors were set up in the areas like the patient entrance, toilet, and other areas to avoid contact with the contact infection. While in partial areas, for the convenience of isolating confirmed patients and promptly sending the patients to wards, airtight doors were added to avoid droplet transmission and were equipped with isolation chambers. The arrangement of waiting chairs should be kept at a safe distance. In addition, traffic signs for the fever clinic were set up in the main entrance of the hospital to guide patients to the clinic to avoid crossing with other people. Fig. 4 shows the medical staff and patient streamlining of the fever clinic after modification.

\section{Sewage treatment and renovation}

With advances in research, positive nucleic acid of the novel coronavirus was found in the feces of patients diagnosed with pneumonia, and there might be viable novel coronavirus in the feces, thus increasing the risk of fecal-oral transmission $[5,6]$. Therefore, there were significant implications regarding sewage treatment discharge from the hospital reaching standards for environmental protection and control of disease spread. Zhijiang campus was designed and built in accordance with the standards of comprehensive hospitals rather than infectious disease hospitals, and the sewage discharge standard of the infectious disease hospital was higher than the general hospital, which had to remove the sewage treatment system urgently as follows: the pretreatment setting of sludge wastewater was added in the front section for wards and clinics related to infectious diseases; the amount of chlorine in the end of the disinfection tank and the standing time of chlorine were increased; and there was continuous follow-up of sampling to ensure that the 
Fig. 4 Streamlining of the fever clinic medical staff and patients

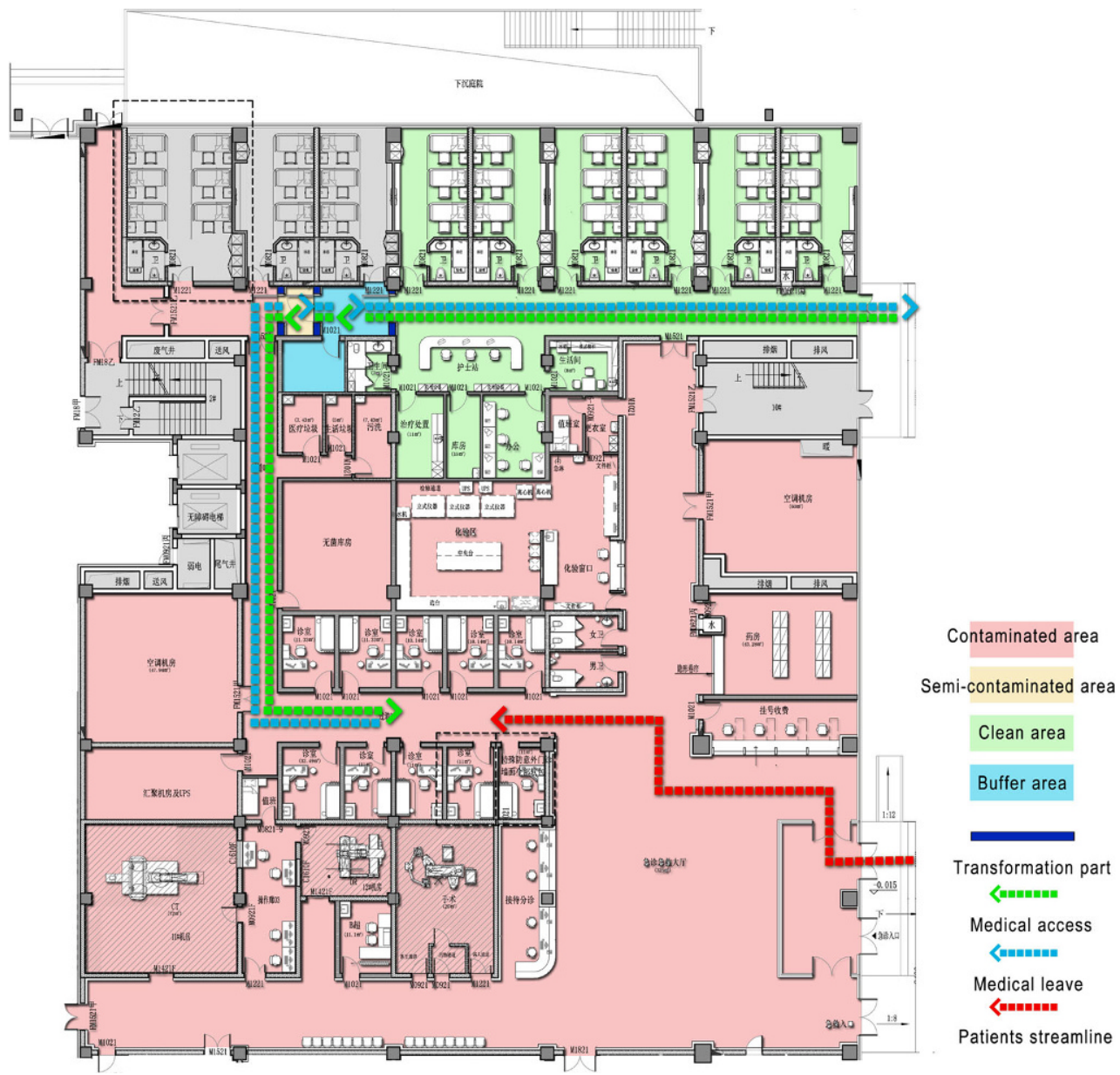

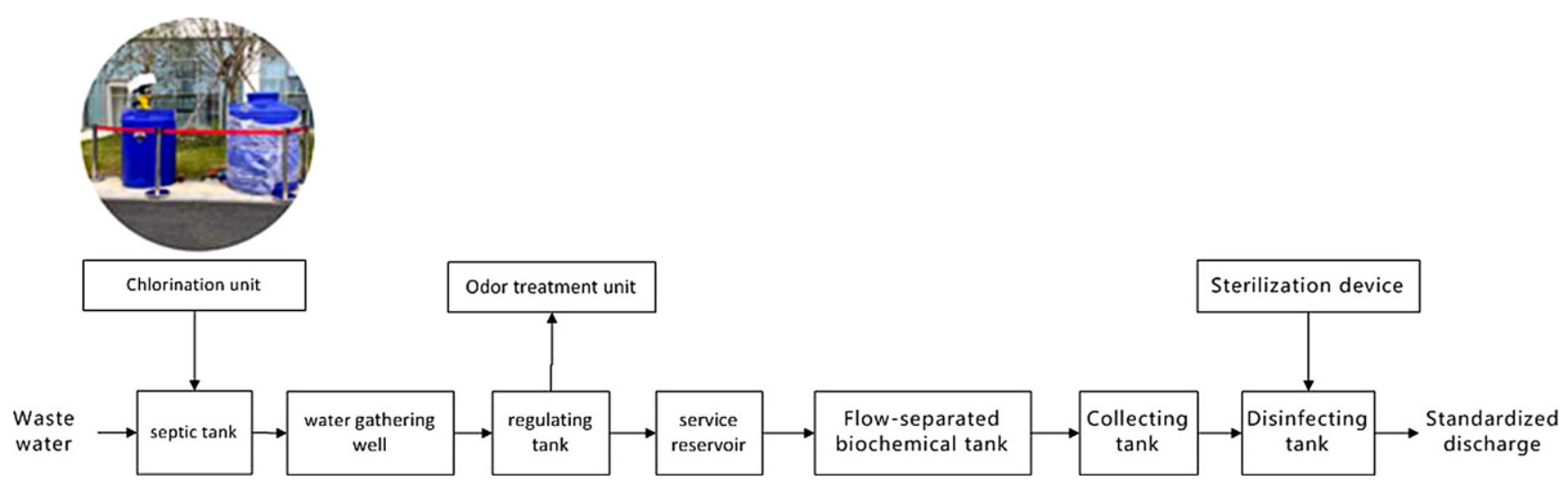

Fig. 5 Sewage treatment flow

discharge water did not contain pathogenic viruses (Fig. 5).

\section{Others}

Transmission by respiratory droplets and contact are the main transmission routes for the new coronavirus [7-11]. According to the requirements of the infectious disease hospital, attention must be paid to the design of the air conditioning and ventilation system of the air flow organization, the air conditioning of clean, semi-contaminated, and contaminated areas, the independent setting of the ventilation system, the relative negative pressure environment must be assured by directing the supply air flow from the clean area to the semi-contaminated area, then to the contaminated area through the pressure difference, and the exhaust air rate must be higher than the air output rate of each area. Because the Zhijiang campus is a general hospital, it does not meet the above require- 
ments. Therefore, to block all possible transmission routes, the central air conditioning, fresh air system, logistics vehicle, and other systems in the Zhijiang campus stopped running.

\section{Discussion}

Due to the complex, highly contagious, close relationship with animals, and high fatality rate, new infectious diseases pose a serious threat to safety, economic development, and social stability [12, 13]. In December 2019, there was a COVID-19 outbreak in Wuhan, China. The main transmission routes were respiratory tract droplet and contact transmission. The main symptoms include fever, fatigue, dry cough, and dyspnea after infection [14-17]. This outbreak of COVID-19 had the characteristics of a sudden, uncertain, and urgent, unprecedented crisis to the hospitals, and also exposed the shortages of prevention and preparation of the city from several aspects with respect to the sudden outbreak of an infectious disease $[18,19]$. For example, in the early period of the outbreak, there were insufficient fever clinics and infectious disease beds, and many patients could not be hospitalized, which led to treatment delays and further spread of the outbreak. The early-stage patients in the hospital were not adequately separated and isolated, which then caused the infection of ordinary patients and medical staff. All of these incidents reminded us that we must focus on the emergency response to outbreaks of infectious diseases from the perspective of the infrastructure of medical institutions.

To solve the shortage of medical places in emergencies, it is common practice to convert public facilities, such as a stadium, exhibition center, and schools as public health emergency facilities, [20] and it is also a feasible plan to temporarily transform general hospitals into infectious disease hospitals. At the beginning of the outbreak, Zhijiang campus was considered for hospital infection control and emergency reconstruction, so the design of a modular triage unit was adopted. Each triage unit had an independent entrance and exit and vertical traffic, which provided technical feasibility for setting up temporary isolation diagnosis area during the outbreak of the infectious disease. Due to the specificity of infectious disease treatment, the space design requirements for the building, which differs from the general hospitals, was put forward. Thus, during the process of emergency transformation from general hospital into an infectious disease hospital, it was necessary to solve a series of problems about how to control the source of infection, cut off the transmission chain, and segregate the susceptible, such as the work area layout, in and out of lines, isolation mode, medical supplies, including the delivery and distribution of food and drugs, pollutants, and indoor air distribution. The improvement of streamlining mainly focused on hos- pital infection control, which proposed that the medical streamline was safe and independent, the medical streamline was efficient and easy to identify, and the cleaning and pollution streamline was one-way and controllable by taking different streamline carriers as objects. The air conditioning and ventilation systems in the clean, semi-contaminated, and contaminated areas were set up independently. The exhaust air volume in each area was greater than the air supply volume to ensure the relative negative pressure environment. If the transformation could not be completed in a short time, the possibility of droplet transmission was considered and the air conditioning and ventilation systems were shut down. Because the infectious ward involved treatment based on isolation, it was necessary to fully consider the psychological relief of the patients. The infectious ward was also equipped with video visitation and telemedicine facilities to facilitate the consultation inside and outside the isolation warehouse.

Since the first confirmed case was admitted to the hospital in Hangzhou, the capital of the Zhejiang province on 19 January, a total of 291 cases had been admitted to the hospital by 10:00 a.m. on 5 March 2020. Among these cases, 71 confirmed cases (all severe and critical) were transferred from other hospitals (95\% critical patients of the entire province). Our hospital admitted 220 suspected cases, including 33 confirmed cases and 187 suspected exclusion discharged cases. Of 5551 fever cases, 104 patients were confirmed, and 95 patients were cured and discharged, among which the oldest was 96 years of age and the youngest was 13 years of age. During the period, a healthy boy was born in a negative pressure operating room to a 35-week pregnant severely ill female patient, and the world's first lung transplantation was performed on a 66-year-old female patient. During the period from 24-30 April 2020, the entire medical staff $(>6500)$ in the hospital underwent RNA testing for SARS-CoV-2 in throat swabs and blood sampling (lgG and $\operatorname{lgM})$. The test results showed that none of the medical staff had positive nucleic acid testing or specific lgG and lgM titers. The FAHZU achieved the goals of zero infection for medical staff, zero missed diagnosis for infection cases, and zero deaths for patients. Through the successful transformation of the Zhijiang campus, it was fully prepared for the possible escalation of the epidemic. On the afternoon of 26 January 2020, all of the patients in the main campus of the FAHZU were transferred to the Zhijiang campus by negative-pressure ambulances. After that, the severe patients in various regions of Zhejiang province were transferred to the Zhijiang campus by ambulance, and the designated treatment work was carried out smoothly.

Despite $95 \%$ of the severe cases from the entire province being admitted to the hospital, none of the medical staff were infected among 6500-not even a single out of hospital case. Relative to the litera- 
ture report of COVID-19 patients with a 2.2-2.3\% case fatality rate, $[21,22]$ the data of the FAHZU was clearly better than the national average, which explained the timely crisis management, correct measures, and successful display of the hospital image, also provided a valuable experience for large medical institutions on how to deal with public health emergencies and how to carry out diagnosis and treatment space, streamline layout, and related facilities in emergency reconstruction; however, in responding to the epidemic in Zhijiang campus, there were also some problems that were difficult to solve through the later transformation. For example, there was no independent setting of air conditioning and the ventilation system in clean, semi-contaminated, and contaminated areas, which led to shutting down the central air conditioning and enabling independent heating equipment during emergencies, which also led to an excessive electricity load. Therefore, we suggest that hospital managers consider problems in the process of the new hospital design, such as a general comprehensive hospital to deal with sudden or new infectious diseases, setting up emergency-enabled doors and independent air conditioning and ventilation systems in clean area, semi-contaminated, and contaminated areas under an epidemic situation, and creation of common wards to admit patients, with rapid conversion into infectious disease wards at the time of the outbreak. Furthermore, the planning of the fever clinics in general hospitals should focus on the combination of peace time and war time design. Under the premise of ensuring basic functions, emergency space should be reserved. For example, fever clinics should reserve outdoor emergency sites, which can be used as temporary hospitals or mobile cabin hospitals in the case of an epidemic outbreak.

Acknowledgements We would like to thank all the participants for their enthusiasm and interest in building a safer healthcare. We would also like to thank Han Zhou for support.

Funding This study was funded by the Science Technology Department of Zhejiang Province, Zhejiang provincial government and Zhejiang University. The funding bodies had no roles in the design of the study, data collection, analysis, interpretation of data, or writing the manuscript.

\section{Compliance with ethical guidelines}

Conflict of interest Y. Chen, M. Zhou, L. Hu, X. Liu, L. Zhuo and Q. Xie declare that they have no competing interests.

Ethical standards The ethics committee of FAHZU reviewed and approved the study. No animal experiments and human trials are involved.

\section{References}

1. Zhu N, Zhang D, Wang W, Li X, Yang B, Song J, et al. A novel coronavirus from patients with pneumonia in China, 2019. NEngl J Med. 2020;382:727-33.

2. Holshue ML, DeBolt C, Lindquist S, Lofy KH, Wiesman J, Bruce H, et al. First case of 2019 novel coronavirus in the United States. NEngl J Med. 2020;382:929-36.

3. Munster VJ, Koopmans M, van Doremalen N, van Riel D, de Wit E. A novel coronavirus emerging in China-key questions for impact assessment. NEngl J Med. 2020;382:692-4.

4. ArashiroT, FurukawaK, NakamuraA.COVID-19in2 persons with mild upper respiratory symptoms on a cruise ship, Japan. Emerg Infect Dis. 2020; https://doi.org/10.3201/ eid2606.200452.

5. Rothe C, Schunk M, Sothmann P, Bretzel G, Froeschl G, Wallrauch C, et al. Transmission of 2019-nCoV infection from an asymptomatic contact in Germany. N Engl J Med. 2020;382:970-1.

6. Xu K, Cai H, Shen Y, Ni Q, Chen Y, Hu S, et al. Management of corona virus disease-19 (COVID-19): the Zhejiang experience. Zhejiang DaXueXue Bao YiXue Ban. 2020;49:0.

7. Rasmussen SA, Smulian JC, Lednicky JA, Wen TS, Jamieson DJ. Coronavirus disease 2019 (COVID-19) and pregnancy: what obstetricians need to know. Am J Obstet Gynecol. 2020; https://doi.org/10.1016/j.ajog.2020.02.017.

8. Li Z, Yi Y, Luo X, Xiong N, Liu Y, Li S, et al. Development and clinical application of A rapid IgM-IgG combined antibody test for SARS-CoV-2 infection diagnosis. J Med Virol. 2020; https://doi.org/10.1002/jmv.25727.

9. Li X, Zai J, Zhao Q, Nie Q, Li Y, Foley BT, et al. Evolutionary history, potential intermediate animal host, and crossspecies analyses of SARS-CoV-2. J Med Virol. 2020; https:// doi.org/10.1002/jmv.25731.

10. Haider N, Yavlinsky A, Simons D, Osman AY, Ntoumi F, ZumlaA, etal. Passengers' destinations from China: lowrisk of novel coronavirus (2019-nCoV) transmission into Africa and South America. Epidemiol Infect. 2020;148:e41.

11. Gao QY, Chen YX, Fang JY. 2019 novel coronavirus infection and gastrointestinal tract. J Dig Dis. 2020; https://doi.org/ 10.1111/1751-2980.12851.

12. Sun P, LuX, Xu C, Sun W, Pan B. Understanding of COVID-19 based on current evidence. J Med Virol. 2020; https://doi. org/10.1002/jmv.25722.

13. Lai CC, Shih TP, Ko WC, Tang HJ, Hsueh PR. Severe acute respiratory syndrome coronavirus 2 (SARS-CoV-2) and coronavirus disease-2019 (COVID-19): the epidemic and the challenges. Int J Antimicrob Agents. 2020;55:105924.

14. Huang C, Wang Y, Li X, Ren L, Zhao J, Hu Y, et al. Clinical features of patients infected with 2019 novel coronavirus in Wuhan, China. Lancet. 2020;395:497-506.

15. Chen N, Zhou M, Dong X, Qu J, Gong F, Han Y, et al. Epidemiological and clinical characteristics of 99 cases of 2019 novel coronavirus pneumonia in Wuhan, China: a descriptive study. Lancet. 2020;395:507-13.

16. Wang D, Hu B, Hu C, Zhu F, Liu X, Zhang J, et al. Clinical characteristics of 138 hospitalized patients with 2019 novel coronavirus-infected pneumonia in Wuhan, China. JAMA. 2020; https://doi.org/10.1001/jama.2020.1585.

17. ChenJ.Pathogenicity and transmissibility of $2019-\mathrm{nCoV}-\mathrm{a}$ quick overview and comparison with other emerging viruses. Microbes Infect. 2020;22:69-71.

18. ArabiYM, MurthyS, WebbS.COVID-19: a novel coronavirus and a novel challenge for critical care. Intensive Care Med. 2020; https://doi.org/10.1007/s00134-020-05955-1.

19. Lee PI, Hsueh PR. Emerging threats from zoonotic coronaviruses-from SARS and MERS to 2019-nCoV. J Microbiol 
Immunol Infect. 2020; https://doi.org/10.1016/j.jmii.2020. 02.001 .

20. Koh HK, Elqura LJ, Judge CM, Stoto MA. Regionalization of local public health systems in the era of preparedness. Annu Rev Public Health. 2008;29:205-18. Review.

21. Bassetti M, Vena A, Giacobbe DR. The novel Chinese coronavirus (2019-nCoV) infections: challenges for fighting the storm. Eur JClin Invest. 2020;50:e13209.
22. She J, Jiang J, Ye L, Hu L, Bai C, SongY.2019 novel coronavirus of pneumonia in Wuhan, China: emerging attack and management strategies. Clin Transl Med. 2020;9:19.

Publisher's Note Springer Nature remains neutral with regard to jurisdictional claims in published maps and institutional affiliations. 